\title{
Air Gauge Characteristics Linearity Improvement
}

\author{
Cz. J. Jermak, M. Jakubowicz, J. Dereżyński, and M. Rucki \\ Institute of Mechanical Technology, Poznań University of Technology, Piotrowo Street 3, 60-965 Poznań, Poland \\ Correspondence should be addressed to M. Rucki; miroslaw.rucki@gmail.com
}

Received 23 November 2015; Revised 14 March 2016; Accepted 20 March 2016

Academic Editor: Carlos Andrés Garcia

Copyright (C) 2016 Cz. J. Jermak et al. This is an open access article distributed under the Creative Commons Attribution License, which permits unrestricted use, distribution, and reproduction in any medium, provided the original work is properly cited.

\begin{abstract}
This paper discusses calibration uncertainty and linearity issues of the typical back-pressure air gauge. In this sort of air gauge, the correlation between the measured dimension (represented by the slot width) and the air pressure in the measuring chamber is used in a proportional range. However, when high linearity is required (e.g., nonlinearity less than 1\%), the measuring range should be shortened. In the proposed method, based on knowledge of the static characteristics of air gauges, the measuring range is kept unchanged but the nonlinearity is decreased. The static characteristics may be separated into two sections, each of them approximated with a different linear function. As a result, the nonlinearity is reduced from $5 \%$ down to $1 \%$ and even below.
\end{abstract}

\section{Introduction}

Air gauges are well-known precise measuring devices [1], which have recently regained the interest of engineers [2]. Even though the definition of the precise machining and demands on the precise measurement are changing due to the development of new methods [3], air gauges are still considered to be high-accuracy measuring devices [4]. They are especially useful in automatic measurement and selection systems [5] and in in-process control [6]. It is assumed that the measurement accuracy of an air gauge is ca. $2 \mu \mathrm{m}$ in the range of $0.001 \mathrm{~mm}$ [7], but in some applications accuracy below $1 \mu \mathrm{m}$ is achievable [8]. Due to their merits, air gauges may replace other measuring devices [9].

The air gauging principle has been known for almost a century. The earliest known patent of this device was registered in the USA in 1922, but the first marketed air gauge was a back-pressure gauge which Solex in Germany introduced in 1926 and Sheffield in the USA in 1935 [10]. The main air gauging methods are flow (velocity) and pressure (backpressure) ones [11], which are in turn divided into highpressure and low-pressure devices. In any case, the measured dimension represented by the flapper surface works as a restriction for the air outflow which influences an air flow parameter. For instance, if the clearance between the nozzle and measured detail surface becomes wider, the mass flow (or velocity) through the air gauge becomes larger, while the back-pressure gets smaller. The magnification of backpressure varies from 1000:1 to over 5000:1 depending on the range, while a flow gauge can amplify to over 500,000:1 without accessories [12].

Nowadays, the dimensional accuracy of any manufacturing process became critical because of increased quality demands. That means the constant need for development of more accurate measuring methods and devices and minimization of expenses (the price of the equipment, as well as operation time and exploitation costs). Air gauges generally meet those requirements, and they can be improved with small additional costs.

Since there is still some room for improvement [13], investigations on air gauges are being performed continuously. The most recently published article gives information concerning the application of back-pressure air gauges in roundness assessment [14] where the calculations and simulations of the static characteristics are based on second critical parameters. However, in typical devices, the problem is with the initial adjustment [15] which is performed usually after each exchange of the jet plug (measuring head) with one or two master rings [16]. Thus two points are set for the linear approximation of static characteristics used further in the measurement. Obviously, shorter segments of the characteristics are more linear, but the proper measuring range should be kept. More calibration points would require more respective master rings, which would increase expenses 

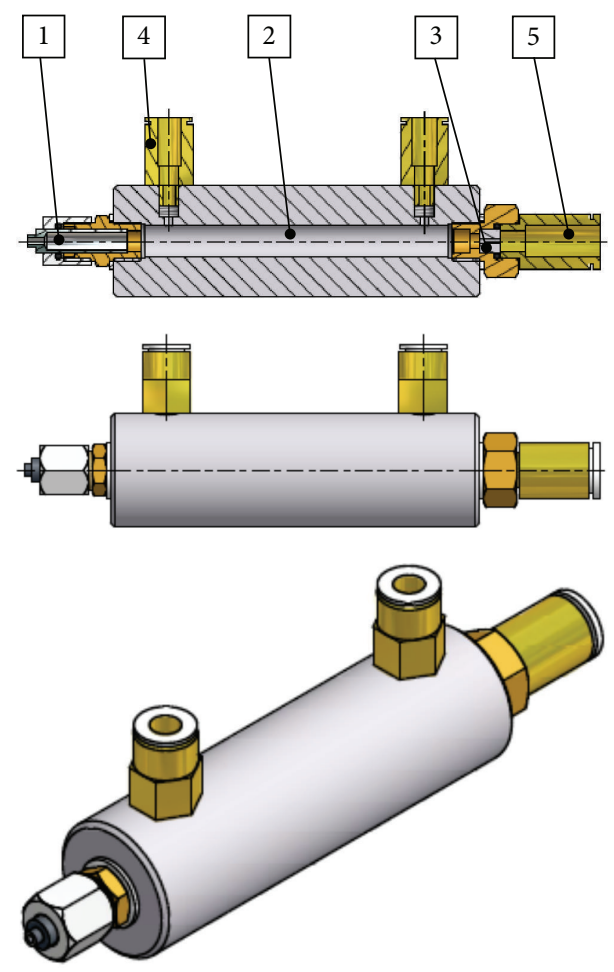

FIGURE 1: Investigation model of the air gauge: 1, replaceable measuring nozzle; 2 , measuring chamber; 3 , replaceable inlet nozzle; 4 , joint for back-pressure sensor; 5 , feeding joint.

significantly, deleting low costs as the main merit of air gauging.

Hence, the state of the art is as follows: the static characteristics of the back-pressure air gauge can be calculated and simulated with high accuracy, but its nonlinearity in a larger measuring range may affect the measurement results. The adjustment process requires expensive master rings, so the number of calibration points should be minimal (typically, two). The proposed method improves the measurement accuracy using two approximation lines and reducing the overall nonlinearity calculated as a difference between the nominal point on the theoretical line and the actual point registered by the device.

\section{Investigation Apparatus}

A group of high-pressure air gauges with the feeding pressure $150 \mathrm{kPa}$ has been investigated for many years in Poznań University of Technology [17]. The investigation model of the air gauge is presented in Figure 1, which enables the changing of the main parameters of an air gauge in order to check its metrological properties experimentally. There are measuring chambers of different dimensions with two or more joints for back-pressure $p_{k}$ measurement, replaceable measuring nozzles with various diameters $d_{p}$ and different geometry (e.g., conical inlet and corrected nozzle head), and replaceable inlet nozzles of diameters $d_{w}$.

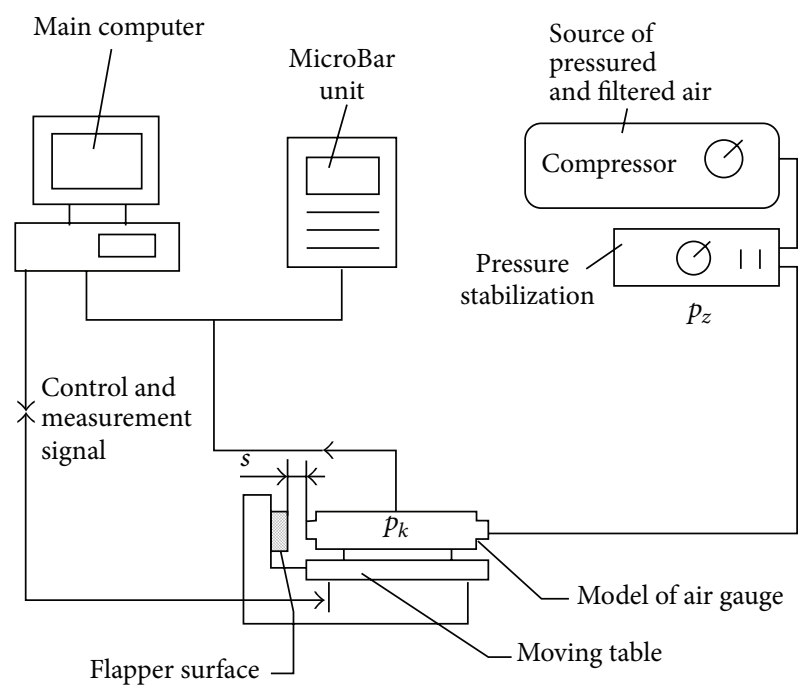

FIGURE 2: Scheme of the static characteristics investigation set [18].

To obtain the static characteristics of an air gauge, the back-pressure signal $p_{k}$ is being compared with the displacement $s$ indication of measuring column TT 500 connected with the inductive sensor GT21HP and analyzed by dedicated software. The laboratory set has been described in detail in [18] and its principle is shown in Figure 2. Additional devices could be included like a MicroBar unit which registers fluctuations of the back-pressure $p_{k}$ with high-frequency sampling (from $16 \mathrm{~Hz}$ up to $4 \mathrm{kHz}$ ) [15] or temperature sensors and flowmeter.

The measuring slot $s$ is slowly changed in the range from 0 up to the maximal value $s_{\max }$ defined by the user, dependent on the examined air gauge. Typically $s_{\max }$ does not exceed 250 or $300 \mu \mathrm{m}$. The registered functions of the back-pressure $p_{k}$, volumetric flow $q_{v}$, and temperatures in the air gauge measuring chamber $t_{1}$ and in the feeding chamber $t_{2}$ could be presented in the following form:

$$
\begin{aligned}
& p_{k \rightarrow}=f(s), \\
& q_{v \rightarrow}=f(s), \\
& t_{1 p \rightarrow}=f(s), \\
& t_{2 p \rightarrow}=f(s), \\
& p_{k \leftarrow}=f(s), \\
& q_{v \leftarrow}=f(s), \\
& t_{1 p \leftarrow}=f(s), \\
& t_{2 p \leftarrow}=f(s),
\end{aligned}
$$

The first set of (1) represents the results obtained from the increasing values of $s$ (the moving table is displacing air 


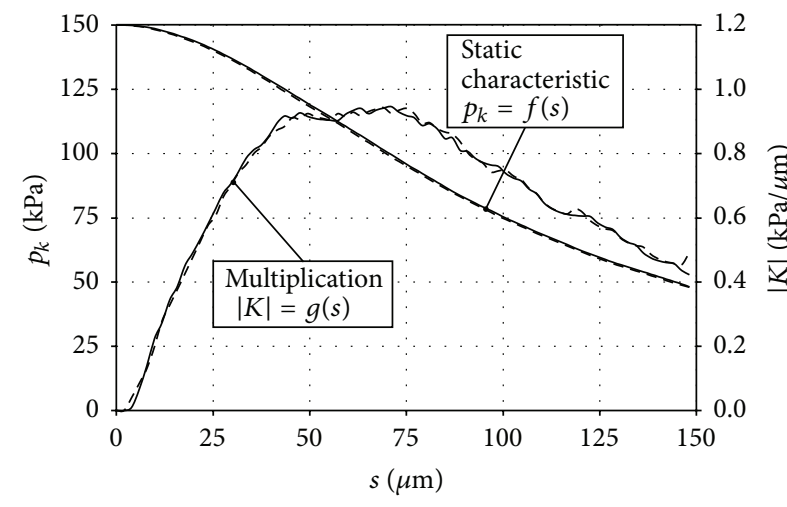

(a)

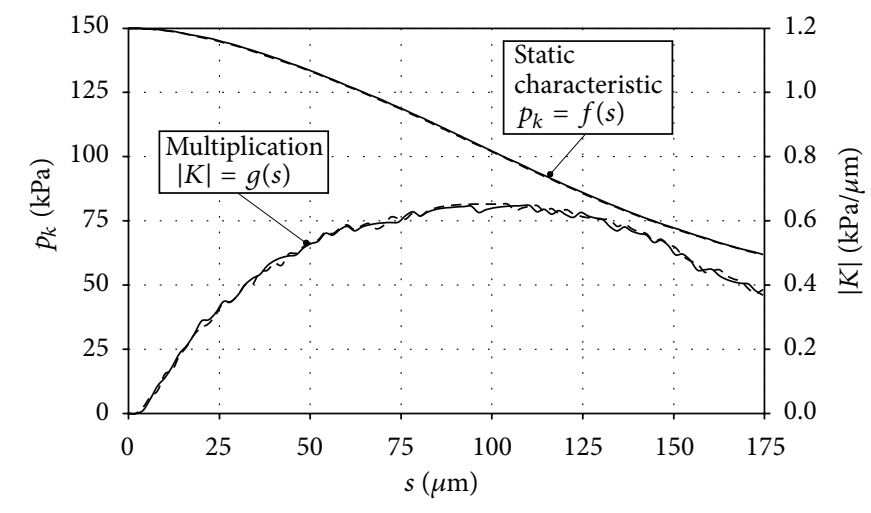

(b)

FIGURE 3: Examples of the experimentally obtained static characteristics and the multiplication graphs of air gauges.

gauge away from the flapper surface) and (2) for decreasing ones, respectively. It is obvious that the collected data contain some noise, so the range of the mathematical functions should be applied to filter metrologically significant information. Thus, the data processing consists of the following steps:

(i) Data smoothing.

(ii) Interpolation.

(iii) Calculation of the multiplication.

(iv) Linearization of the experimentally obtained function $p_{k}=f(s)$.

After the data is processed, the result may be presented both for average values and for separate increasing and decreasing functions. The latter enables the possibility of revealing a hysteresis.

From the numerous smoothing methods (see, e.g., [19]) the one based on the least squares has been chosen. On the basis of the subsequent $2 n+1$ values of the variable (see (3)), the polynomial of $j$ degree (see (4)) is fitted to achieve the minimal differences between the calculated values $y\left(x_{i}\right)$ and measured ones $y_{i}$ :

$$
\begin{gathered}
y_{i-n}, y_{i-n}, \ldots, y_{i}, \ldots, y_{i+n-1}, \ldots, y_{i+n}, \\
y(x)=c_{0}+c_{1} x+c_{2} x^{2}+\cdots+c_{j} x^{j}, \\
\sum_{k=-n}^{n}\left[y_{i+k}-y\left(x_{i+k}\right)^{2}\right]=\min .
\end{gathered}
$$

The values $y\left(x_{i}\right)$ obtained in this way are assumed to be the values of the smoothened function $y_{i}^{\prime}=y\left(x_{i}\right)$. In the experiments, a quadratic polynomial has been used, based on
7 measured points (see (6)), and the third-degree polynomial based on 9 measured points (see (7)):

$$
\begin{aligned}
& y_{i(2,7)}(x)=\frac{1}{21}\left(-2 y_{i-3}+3 y_{i-2}+6 y_{i-1}+7 y_{i}+6 y_{i+1}\right. \\
& \left.+3 y_{i+2}-2 y_{i-3}\right), \\
& y_{i(3,9)}(x)=\frac{1}{231}\left(-21 y_{i-4}+14 y_{i-3}+39 y_{i-2}+54 y_{i-1}\right. \\
& \left.+59 y_{i}+54 y_{i+1}+39 y_{i+2}+14 y_{i+3}-21 y_{i+4}\right) .
\end{aligned}
$$

It should be noted that, for a higher degree of polynomial, more points should be considered, and the obtained function is less smoothened. The procedure could be repeated several times for the same data, and the number of repetitions could be chosen after the empirical analysis of a series of calculations.

Among many known methods, linear interpolation [20] has been chosen to achieve a steady distribution of the measurement data. It is described by the following formula:

$$
L(x)=y_{0}+\frac{y_{1}-y_{0}}{x_{1}-x_{0}}\left(x-x_{0}\right)
$$

which has appeared sufficient in order to achieve the desired results from the collected data.

To evaluate the linearity of the obtained static characteristics, the multiplication was calculated for each point and the function $|K|=f(s)$ was derived. The function presented graphically gives quick information on the linearity of the analyzed static characteristics $p_{k}=f(s)$ : the multiplication of the linear part of the characteristics keeps at the same level for some time. For example, in Figure 3 one can compare graphs of two experimentally achieved multiplication functions. It is seen that, in one case (a), a linear range from 45 to $75 \mu \mathrm{m}$ could be extracted, and in the other case (b) there is no horizontal part of the multiplication graph; hence, the characteristics reveal worse linearity, though the range between 80 and $120 \mu \mathrm{m}$ may be considered satisfactorily linear. 
Since multiplication is defined as a tangent of the static characteristics declination angle, the local multiplication could be calculated as follows:

$$
K(s)=\left.\frac{d f(s)}{d s} \approx \frac{\Delta p_{k}}{\Delta s}\right|_{s \in(s, s+\Delta s)} .
$$

In a real dataset, however, one deals with discrete values, and then the multiplication should be determined between the points $n+1$ and $n$ :

$$
K_{n}=f(n+1)-\left.f(n)\right|_{n \in(0, z)} .
$$

\section{Calibration Uncertainty}

The calibration methods are described in the standard [21]. To perform the analysis of the air gauge calibration uncertainty, it is useful to present its static characteristics in the following form:

$$
p_{k}=f(s)+\eta=p_{k(s=0)}+K s+\eta,
$$

where $p_{k(s=0)}$ is the starting point of the characteristics, when the measuring nozzle is touching the flapper surface (slot $s=$ $0), K$ is multiplication, and $\eta$ is the uncertainty.

Estimators of those parameters could be calculated from the following equations:

$$
\begin{aligned}
\widehat{p}_{k(s=0)} & =p_{k(s=0)_{s r}}-\widehat{K} s_{s r}, \\
\widehat{K} & =\frac{\sum_{i=1}^{N}\left(s_{i}-s_{s r}\right)\left(p_{k_{i}^{\prime} s^{r}}-p_{k^{\prime} r}\right)}{\sum_{i=1}^{N}\left(s_{i}-s_{s r}\right)^{2}},
\end{aligned}
$$

where $p_{k s}$ and $s_{s r}$ correspond to the mean values:

$$
\begin{gathered}
s_{s r}=\frac{1}{N} \sum_{i=1}^{N} s_{i} ; \\
p_{k \dot{s} r}=\frac{1}{N} \sum_{i=1}^{N} p_{k_{i}} .
\end{gathered}
$$

Thus, the estimation formula will be presented as follows:

$$
\widehat{p}_{k}=\widehat{p}_{k(s=0)}+\widehat{K} s=p_{k^{\prime} s}+\widehat{K}\left(s-s_{s r}\right)
$$

And the calibration characteristics are described by

$$
\widehat{\mathcal{s}}^{*}=c_{0}+c_{1} \widehat{p}_{k}=c_{0}+c_{1}\left(\widehat{p}_{k(s=0)}+\widehat{K} s\right) \text {, }
$$

where

$$
\begin{aligned}
& c_{0}=-\frac{\widehat{p}_{k(s=0)}}{\widehat{K}} ; \\
& c_{1}=\frac{1}{\widehat{K}} .
\end{aligned}
$$

The assumptions for the calibration procedure were as follows:

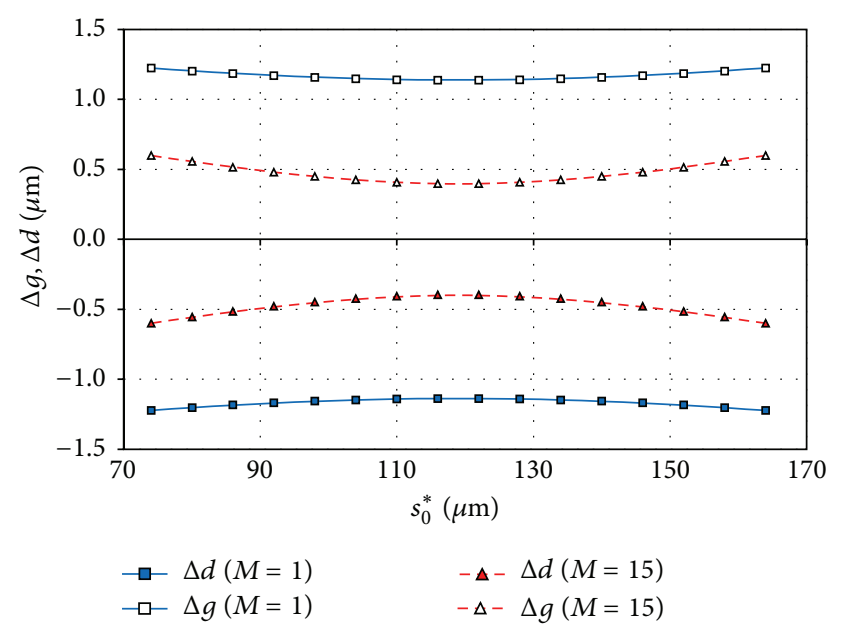

Figure 4: Calibration uncertainties for air gauges with measuring

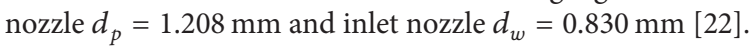

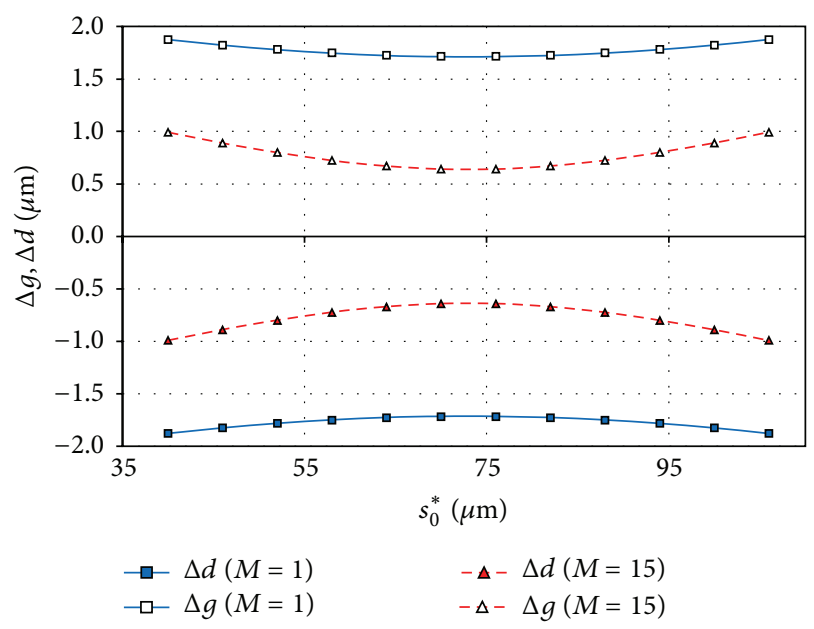

FIGURE 5: Calibration uncertainties for air gauges with measuring

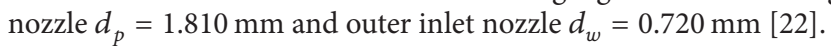

(i) Expected value of $\eta$ is zero $(E\{\eta\}=0)$.

(ii) Variation of $\eta$ is constant and independent of $s$ $\left(\operatorname{var}\{\eta\}=\sigma_{\eta}^{2}\right)$.

(iii) The probability distribution of the random errors is Gaussian $\left(p(\eta)=N\left(0, \sigma_{\eta}^{2}\right)\right)$.

(iv) Errors are not correlated with the measured value $s$ $(\operatorname{cov}\{\eta, s\}=0)$.

(v) The errors of the set masters are negligibly small.

When the same value of $s_{0}$ in the process $\left\{\eta_{0}\right\}$ is measured $M$ times, the uncertainty of the calibrated system could be determined as the confidence interval $\Delta=s^{*}-s$. The examples of upper $(\Delta g)$ and lower $(\Delta d)$ confidence intervals for $M=1$ and $M=15$ are presented in the graphs (Figures 4 and 5). 
TABLE 1: Results of the calibration.

\begin{tabular}{lccccccc}
\hline Parameter & $s_{p}=40 \mu \mathrm{m}$ & $s_{p}=50 \mu \mathrm{m}$ & $s_{p}=60 \mu \mathrm{m}$ & $s_{p}=70 \mu \mathrm{m}$ & $s_{p}=80 \mu \mathrm{m}$ & $s_{p}=90 \mu \mathrm{m}$ & $s_{p}=100 \mu \mathrm{m}$ \\
\hline$s_{k}[\mu \mathrm{m}]$ & 91.6 & 113.5 & 139.5 & 165.5 & 191.5 & 221.5 & 249.5 \\
Measuring range $z_{p}[\mu \mathrm{m}]$ & 52.1 & 63.9 & 79.9 & 96.0 & 111.9 & 134.0 & 149.8 \\
$R^{2}$ & 0.9978 & 0.9991 & 0.9984 & 0.9969 & 0.9953 & 0.9922 & 0.9902 \\
$r$ & 0.9989 & 0.9995 & 0.9992 & 0.9984 & 0.9976 & 0.9961 & 0.9951 \\
$\delta_{\max }[\%]$ & 2.9 & 2.0 & 3.6 & 4.2 & 5.4 & 6.5 & 4.8 \\
\hline
\end{tabular}

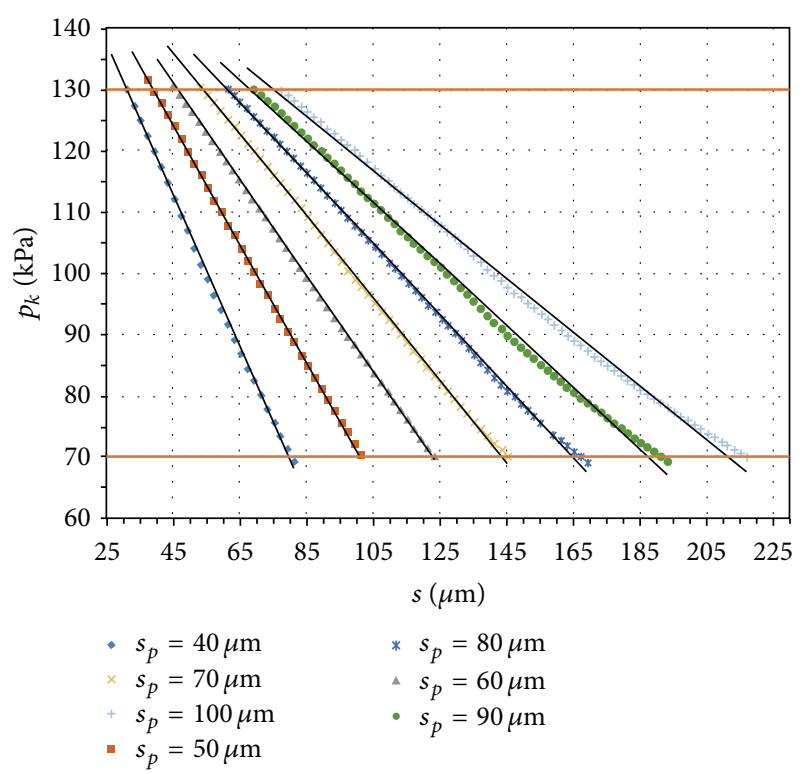

FIGURE 6: Linear approximation of the air gauge characteristics in the pressure range between 120 and $60 \mathrm{kPa}$.

\section{Linearity Improvement}

Earlier investigations had proved that it is possible to achieve better linearity by applying more setting masters [23]. More recent investigations were made in Poznań University of Technology to improve linearity in the air gauging system Pneusmart. The system includes a mechanical unit (gauging heads, etc.), a pneumatic unit, and a control unit [24], and linearity is improved digitally by dividing the measuring range into two parts.

In fact, the approximation of the static characteristics in the required measuring range could give too poor linearity. Table 1 contains the results of measurement and calculations, which correspond to graphs in Figure 6. Here $s_{p}$ is the slot width at which the measuring range $z_{p}$ begins, and $s_{k}$ is the one it ends with. $R^{2}$ is the determination factor which given in percentage shows how many points are determined by the regression [25]. $R^{2}$ is corresponding to the correlation factor $r$ :

$$
r=\sqrt{R^{2}}
$$

It is assumed that when the correlation factor $r$ lies between 0.9 and 1 , the correlation is almost complete, which is the case shown in Table 1.

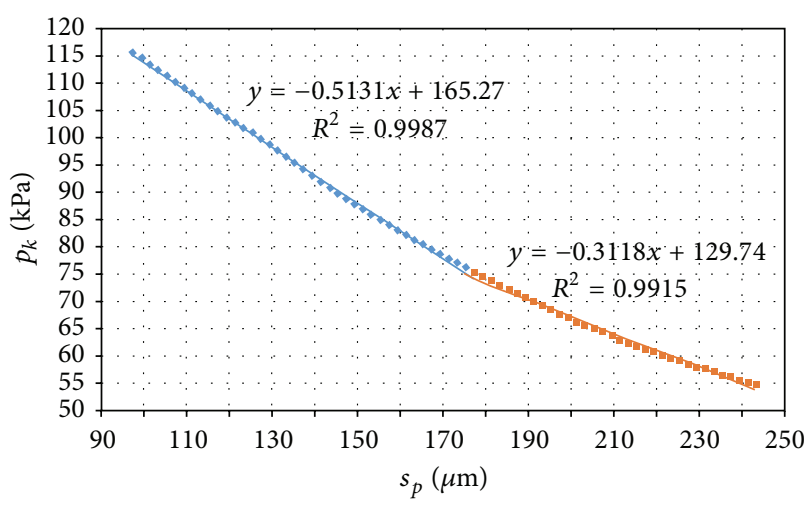

- Section A approximation

- Section B approximation

FIgURE 7: Example of the characteristics approximated with two functions.

However, the linearity error from $2 \%$ to $6.5 \%$ is highly unsatisfactory. To reduce the linearity error, the measuring range $z_{p}$ should be shortened. In Figure 7, the static characteristics are presented, which reveal the nonlinearity as more than $2 \%$ in the range $z_{p}$ from 100 to $240 \mu \mathrm{m}$. A reduced measuring range from 100 to $170 \mu \mathrm{m}$ (section A in Figure 7) has the linearity error $\delta_{\max }=0.8 \%$.

Thus, in order to keep the longer measuring range the approximation with two functions is proposed. The static characteristics are then divided into two sections (A and B in the example shown in Figure 7), each approximated with its own function. The maximal linearity error for the entire measuring range $z_{p}$ from 100 to $240 \mu \mathrm{m}$ is reduced down to $\delta_{\max }=0.8 \%$.

The Pneusmart device enables the collection of experimental data on the static characteristics of the particular measuring head (gauge plug) and to keep it in memory. To calibrate it later, it is enough to recall the appropriate characteristics ascribed to this plug and to check just one point with the setting master.

\section{Discussion}

The air flow through the nozzles, measuring chamber, and the flapper-nozzle area itself generates some phenomena impossible to omit, which has been described decades ago [26]. Although some improvement could be made [27] on the curve function $p_{k}=f(s)$ to prolong its proportional (linear) range, the problem stays basically the same: in a 
wider range the linearity of the functions gets worse. An additional problem is the need of adjustment any time the jet plug (measuring head) is changed to perform a different measuring task. It has been found that the adjustment with more than two master rings can improve the measurement results, but the expenses rise disproportionately.

In the previous investigations, due to the electronic conversion of the pneumatic signal and then digital processing of the measured data, it appeared possible to store the onceadjusted function and then to recall it after the exchange of the jet plug. In that case, to calibrate the new jet plug, it is enough to use one setting master to check just one point on the recorded linear characteristics.

The results presented above prove that the next step could be done to reduce the final measurement uncertainty which is affected by the nonlinearity of the $p_{k}=f(s)$ function. The laboratory equipment (Figure 2) enabled the collection of scores of measuring points to check the difference between the actual measurement result and the theoretical linear function obtained in the adjustment process. The obtained results confirmed the well-known rule that better linearity could be achieved in a smaller measuring range. In the case of Figure 7, only the range ca. $70 \mu \mathrm{m}$ is able to provide the acceptable nonlinearity $\delta_{\max }=0.8 \%$ which however appears unacceptably short for many applications.

The Pneusmart device makes it possible to join together two sections of the $p_{k}=f(s)$ function, each of them approximated with a different line and each of them of acceptable nonlinearity $\delta_{\max }=0.8 \%$. The overall measuring range is kept the same, ca. $140 \mu \mathrm{m}$. Then, in an industrial environment, the adjustment could be performed with two master rings, as usual, but the final measurement result will be much less affected by the nonlinearity error of approximation.

\section{Conclusion}

The investigation results led to the following conclusion: new programmable air gauging devices may increase measurement accuracy keeping long measuring range after the appropriate calibration and the approximation with two functions. The linearity error, which appears to be as large as $6 \%$ for long measuring ranges, could be reduced down to $1 \%$, which is a satisfactory result. In this way nonlinearity is not the main factor affecting the measurement uncertainty of an air gauge.

\section{Competing Interests}

The authors declare that there are no competing interests related to this paper.

\section{References}

[1] C. J. Tanner, "Air gauging-history and future developments," Institution of Production Engineers Journal, vol. 37, no. 7, pp. 448-462, 1958.

[2] G. Schuetz, "Pushing the limits of air gaging-and keeping them there," Quality Magazine, vol. 54, no. 7, pp. 22-26, 2015.
[3] N. Taniguchi, "Current status in, and future trends of, ultraprecision machining and ultrafine materials processing," CIRP Annals-Manufacturing Technology, vol. 32, no. 2, pp. 573-582, 1983.

[4] J. Destefani, “Air gaging," Manufacturing Engineering, vol. 131, no. 4, pp. 5-9, 2003.

[5] Y. H. Wang, S. Q. Hu, and Y. Hu, "An automatic sorting system based on pneumatic measurement," Key Engineering Materials, vol. 295-296, pp. 563-568, 2005.

[6] Ch. Koehn, "In-process air gaging," Quality Magazine, vol. 53, no. 5, pp. 22-23, 2014.

[7] W. Gopel, J. Hesse, and J. N. Zemel, Sensors: A Comprehensive Survey, vol. 8, VCH Press, Weinheim, Germany, 1995.

[8] T. Thomas, C. Hamaker, J. Martyniuk, and G. Mirro, "Nanometer-level autofocus air gauge," Precision Engineering, vol. 22, no. 4, pp. 233-242, 1998.

[9] G. Schuetz, "When air blows mechanical gaging away," Quality Magazine, vol. 53, no. 1, pp. 28-32, 2014.

[10] J. A. Bosch, Coordinate Measuring Machines and Systems, Marcel Dekker, Inc, New York, 1995.

[11] M. Curtis and F. Farago, Handbook of Dimensional Measurement, Industrial Press, New York, NY, USA, 2014.

[12] H. F. Walker, A. K. Elshennawy, B. C. Gupta, and M. M. Vaughn, The Certified Quality Inspector Handbook, ASQ Quality Press, Milwaukee, Wis, USA, 2013.

[13] Cz. J. Jermak and M. Rucki, "Air gauging: still some room for development," AASCIT Communication, vol. 2, no. 2, pp. 29-34, 2015.

[14] C. J. Jermak and M. Rucki, "Static characteristics of air gauges applied in the roundness assessment," Metrology and Measurement Systems, vol. 23, no. 1, pp. 85-96, 2016.

[15] M. Rucki, "Reduction of uncertainty in air gauge adjustment process," IEEE Transactions on Instrumentation and Measurement, vol. 58, no. 1, pp. 52-57, 2009.

[16] J. Liu, X. Pan, G. Wang, and A. Chen, "Design and accuracy analysis of pneumatic gauging for form error of spool valve inner hole," Flow Measurement and Instrumentation, vol. 23, no. 1, pp. 26-32, 2012.

[17] Cz. J. Jermak and M. Rucki, Air Gauging: Static and Dynamic Characteristics, IFSA, Barcelona, Spain, 2012.

[18] M. Rucki, B. Barisic, and T. Szalay, "Analysis of air gage inaccuracy caused by flow instability," Measurement, vol. 41, no. 6, pp. 655-661, 2008.

[19] J. S. Simonoff, Smoothing Methods in Statistics, Springer Series in Statistics, Springer, New York, NY, USA, 1996.

[20] W. Dos Passos, Numerical Methods, Algorithms and Tools in C\#, CRC Press, Boca Raton, Fla, USA, 2010.

[21] International Organization for Standardization, "Linear calibration using reference materials," International Standard ISO 11095:1996, International Organization for Standardization, 1996.

[22] M. Jakubowicz and Cz. J. Jermak, "Measurement uncertainty of air guages for length measurement," Machine Engineering, vol. 18, no. 3, pp. 48-59, 2013 (Polish).

[23] M. Rucki, B. Barisic, and G. Varga, "Air gauges as a part of the dimensional inspection systems," Measurement, vol. 43, no. 1, pp. 83-91, 2010.

[24] J. Dereżyński, "PNEUSMART-pneumatic system for geometrical measurements," Machine Engineering, vol. 18, no. 3, pp. 8899, 2013 (Polish). 
[25] M. Ezekiel, Methods of Correlation Analysis, Wiley, New York, NY, USA, 1945.

[26] R. Breitinger, Fehlerquellen beim pneumatischen Längenmessen [Ph.D. thesis], TU Stuttgart, 1969.

[27] C. Crnojevic, G. Roy, A. Bettahar, and P. Florent, "The influence of the regulator diameter and injection nozzle geometry on the flow structure in pneumatic dimensional control systems," Journal of Fluids Engineering, vol. 119, no. 3, pp. 609-615, 1997. 


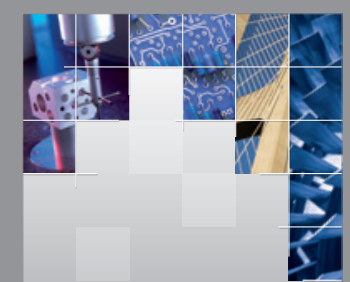

\section{Enfincering}
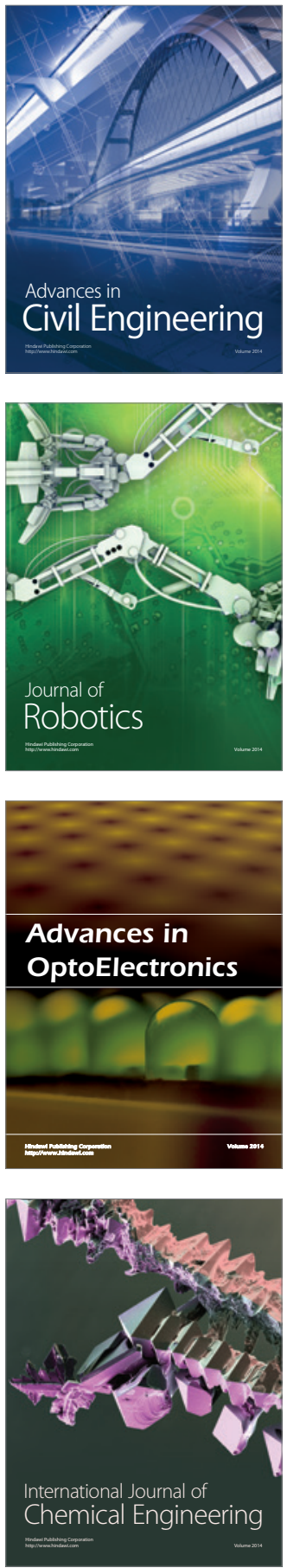

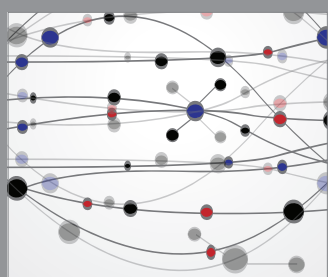

The Scientific World Journal

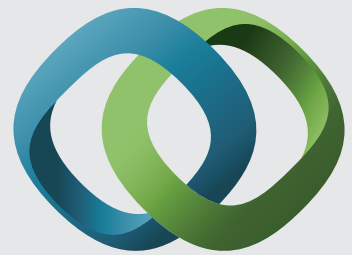

\section{Hindawi}

Submit your manuscripts at

http://www.hindawi.com
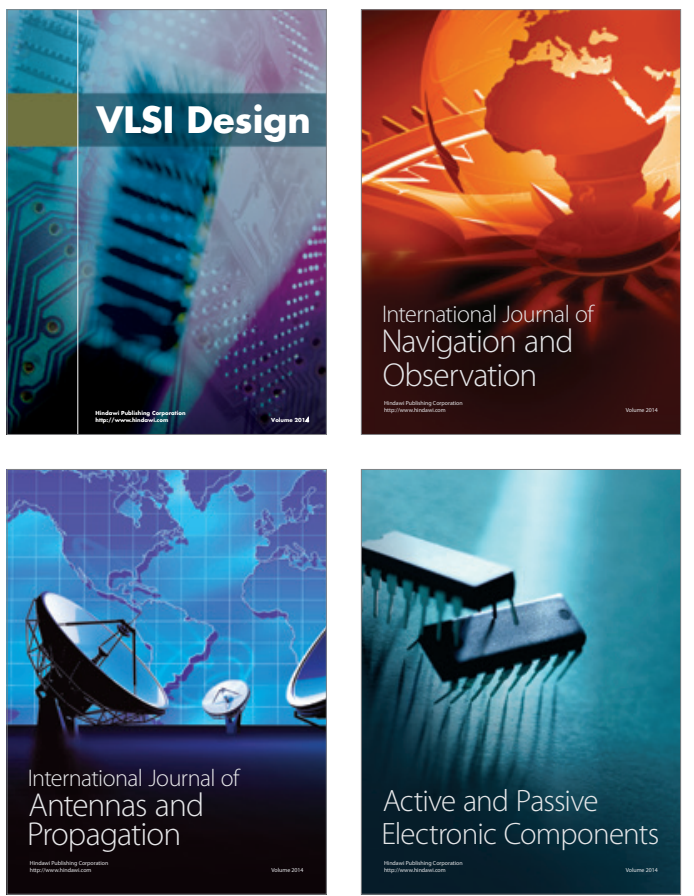
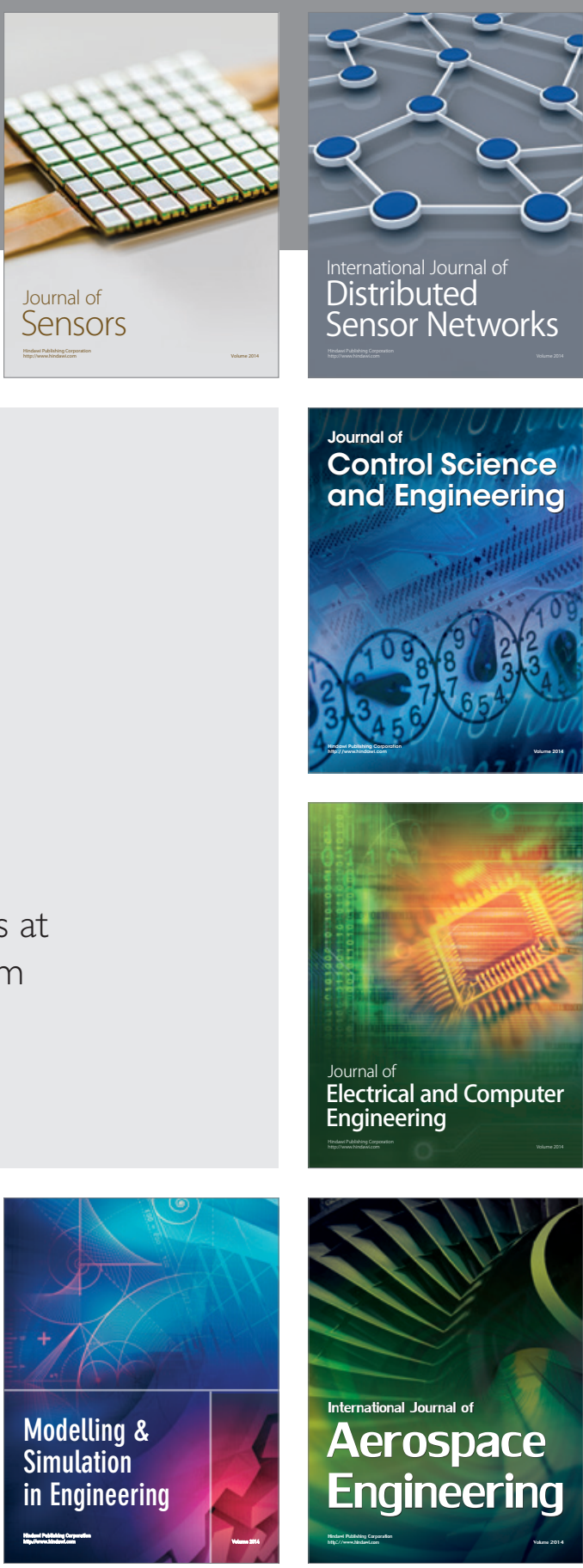

International Journal of

Distributed

Sensor Networks

Journal of

Control Science

and Engineering
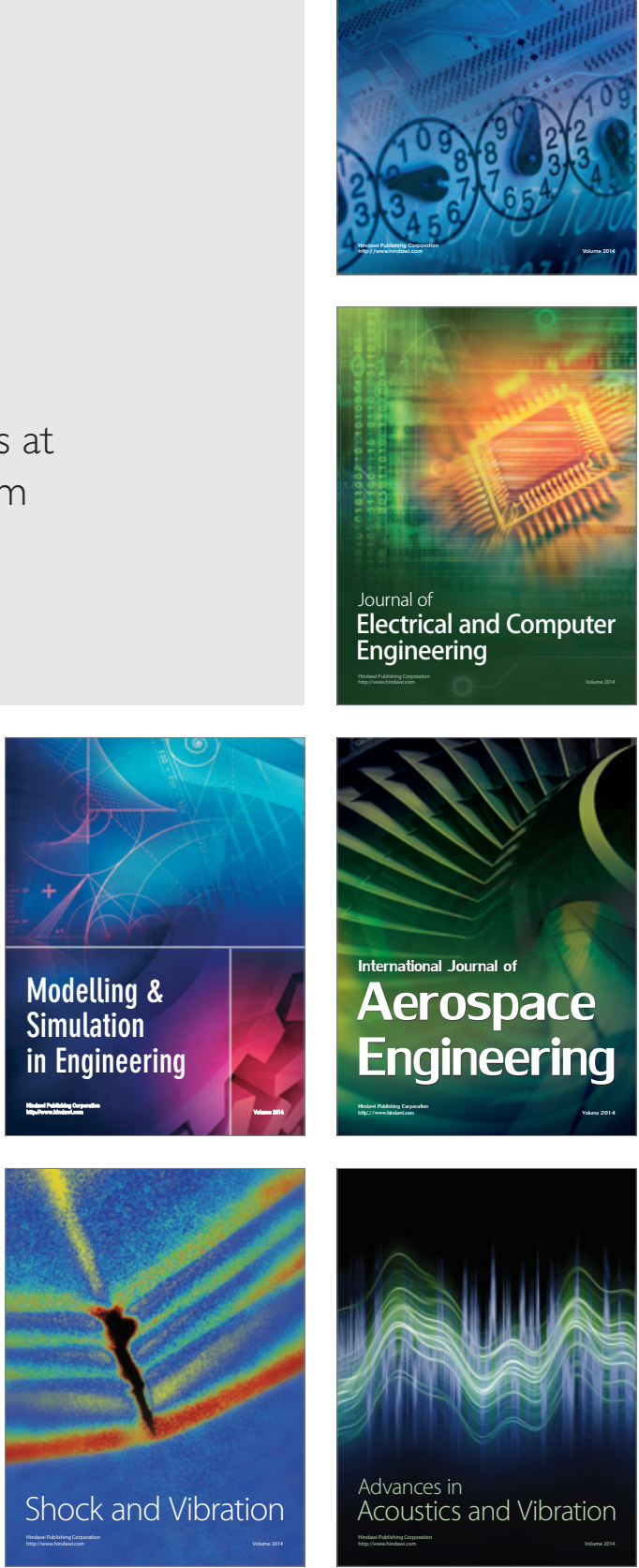